\title{
Nonextensive theory of dark matter and gas density profiles
}

\author{
M. P. Leubner \\ Institute of Astrophysics, University of Innsbruck, A-6020 Innsbruck, Austria \\ manfred.leubner@uibk.ac.at
}

\begin{abstract}
Pronounced core-halo patterns of dark matter and gas density profiles, observed in relaxed galaxies and clusters, were hitherto fitted by empirical power-laws. On the other hand, similar features are well known from astrophysical plasma environments, subject to long-range interactions, modeled in the context of nonextensive entropy generalization. We link nonextensive statistics to the problem of density distributions in large-scale structures and provide fundamentally derived density profiles, representing accurately the characteristics of both, dark matter and hot plasma distributions, as observed or generated in simulations. The bifurcation of the density distribution into a kinetic dark matter and thermodynamic gas branch turns out as natural consequence of the theory and is controlled by a single parameter kappa, measuring physically the degree of coupling within the system. Consequently, it is proposed to favor nonextensive distributions, derived from the fundamental physical context of entropy generalization and accounting for nonlocality and long-range interactions in gravitationally coupled systems, when modeling observed density profiles of astrophysical structures.
\end{abstract}

Subject headings: cosmology:theory — dark matter — galaxies: halos — galaxies:structure — plasmas

\section{Introduction}

The analysis of dark matter (DM) and gas density distributions in galaxies and clusters is presently based on a variety of phenomenological models. Following King (1962) relaxed DM halo density profiles are well fitted by empirical power laws (Burkert 1995; Salucci \& Burkert 2000) and from N-body simulations by $\rho_{D M} \sim\left(r / r_{s}\right)^{-1}(1+$ $r\left(r_{s}\right)^{-2}$ (NFW), where $r_{s}$ constitutes a scaling radius, chosen to join the asymptotic $r$-dependence (Navarro 1996, 1997). Subsequently a number of modifications were proposed (Fukushige \& Makino 1997; Moore et al. 1998, 1999) along with criticism of the 'universality' of the NFW-profile (Jing \& Suto 2000; Borriello \& Salucci 2001). Comparing the density profiles of DM haloes in cold dark matter (CDM) N-body simulations the functional dependence $\rho_{D M} \sim\left(r / r_{s}\right)^{-\alpha}\left(1+r / r_{s}\right)^{-(3-\alpha)}$ (Zhao 1996) was found to provide good fits to all haloes, from dwarf galaxies to clusters at any redshift (Ricotti 2003), where $\alpha$ is related to the spectral index of the initial power spectrum of density perturba- tions. Recently a universal density profile for dark and luminous matter was suggested (Merritt et al. 2005). Physically, we regard the DM halo as a self-gravitating collisionless system of weakly interacting particles in dynamical equilibrium, scale invariant from galactic to cluster scales (Burkert 2000; Firmani et al. 2000; Spergel \& Steinhard 2000).

On the other hand, the phenomenological $\beta$-model $\rho_{\text {gas }} \sim\left(1+r^{2} / r_{c}^{2}\right)^{-3 / 2 \beta}$ (Cavaliere 1976), where $r_{c}$ is the core radius, or the double $\beta$ model, a convolution of two $\beta$-models with the aim of resolving the $\beta$-discrepancy (Bahcall \& Lubin 1994), provide reasonable representations of the hot gas density distribution in galaxies and clusters (Xue \&Wu 2000; Ota \& Mitsuda 2004). Physically, $\beta$ corresponds to the ratio of kinetic $\mathrm{DM}$ and thermal gas energy, assuming values $2 / 3$.

Since any astrophysical system is subject to long-range gravitational or electromagnetic interactions, the present situation motivates to intro- 
duce nonextensive statistics as theoretical basis for both, DM and hot plasma density profiles, utilized successfully to understand observed core-halo structures in astrophysical plasmas (Leubner 2004; Leubner \& Voeroes 2005). In this situation a single parameter $\kappa$ characterizes the degree of nonextensivity or coupling within the system. The corresponding derived power-law distributions constitute a particular thermodynamic equilibrium state (Treumann 1999), commonly applied in astrophysical plasma modeling (Leubner \& Schupfer 2001; Leubner 2002).

The concept of the Boltzmann-Gibbs-Shannon (BGS) thermo-statistics constitutes a powerful tool whenever the physical system is extensive, i.e. the entropy is additive. This situation holds when microscopic interactions and memory are short ranged and the environment is an Euclidean space-time, a continuous and differentiable manifold. However, astrophysical systems are generally subject to spatial or temporal long-range interactions making their behavior nonextensive. A generalization of the BGS entropy for statistical equilibrium was introduced from first principles by Renyi (1955) and Tsallis (1988), suitably extending the standard additivity to nonextensivity. The main theorems of the classical Maxwell-Boltzmann statistics admit profound generalizations within nonextensive statistics leading to a variety of physical consequences, see e.g. Tsallis (1995). Those include a reformulation of the classical N-body problem (Plastino, Plastino, \& Tsallis 1994) or the development of nonextensive distributions (Silva, Plastino, \& Lima 1998; Almeida 2001; Andrade et al. 2002) where the duality of nonextensive statistics, we will focus on, was recognized (Karlin, Grmela \& Gorban 2002). Astrophysical applications (Plastino \& Plastino 1993; Kaniadakis et al. 1996; Nakamichi et al. 2002), provided further manifestation of nonextensivity in nature. For a reformulation in the context of special relativity see Kaniadakis (2002).

\section{Theory}

The generalized entropy $S(\kappa)$ characterizing systems subject to long-range interactions and couplings in nonextensive statistics reads (Tsallis 1988; Leubner 2004)

$$
S_{\kappa}=\kappa k_{B}\left(\sum p_{i}^{1-1 / \kappa}-1\right)
$$

where $p_{i}$ is the probability of the $i^{\text {th }}$ microstate, $k_{B}$ is Boltzmann's constant and the 'entropic index' $\kappa$ denotes a coupling parameter quantifying the degree of nonextensivity, or equivalently statistical correlations, within the system. A crucial property of this entropy is the pseudo-additivity for given subsystems in the sense of factorizability of the microstate probabilities. The transformation $\kappa=1 /(1-q)$ links the $\kappa$-formalism to the Tsallis q-statistics (Leubner 2002). Here $\kappa$ is defined in the interval $-\infty \leq \kappa \leq \infty$ where $\kappa=\infty$ represents the extensive limit of statistical independence and recovers the classical BGS entropy as $S_{B}=-k_{B} \sum p_{i} \ln p_{i}$. Considering two subsystems $A$ and $B$ the nonextensive characteristics can be illuminated in view of the entropy mixing by $S_{\kappa}(A+B)=S_{\kappa}(A)+S_{\kappa}(B)+S_{\kappa}(A) S_{\kappa}(B) / \kappa$, a relation consistent with Eq. (1) where the last term accounts for the couplings. $\kappa<0$ leads to an entropy decrease providing a state of higher order, whereas for $\kappa>0$ the entropy increases and the system evolves into disorder. Hence, $\kappa$ can be interpreted as a bifurcation parameter measuring the two statistical realizations of ordering or disordering through correlations. A generalization for multiple subsystems is discussed by Milovanov \& Zelenyi (2000).

Since entropy and probability distributions reside physically on the same level the corresponding generalized energy distributions follow as $f^{ \pm}(v)=A^{ \pm}\left[1+v^{2} /\left(\kappa \sigma^{2}\right)\right]^{-\kappa}$ (Silva, Plastino, \& Lima 1998; Leubner 2004). The superscripts \pm correspond to positive or negative values of $\kappa$, $A^{ \pm}$are proper normalization constants, and $\sigma$ denotes the velocity dispersion or mean energy of the distribution characterising their width (variance). Negative values of $\kappa$ are conveniently introduced by changing the sign at appearance, which generates a cutoff at $v=\sqrt{\kappa} \sigma$, see Leubner (2004) for details.

Upon generalization to a spherical symmetric, self-gravitating and collisionless N-body system the corresponding steady state phase-space distribution $f(r, v)$ obeys the Vlasov equation. If the particles (stellar system, galaxies) itself provide the gravitational potential and $f(r, v)$ is regarded as the mass distribution then Poisson's equation 


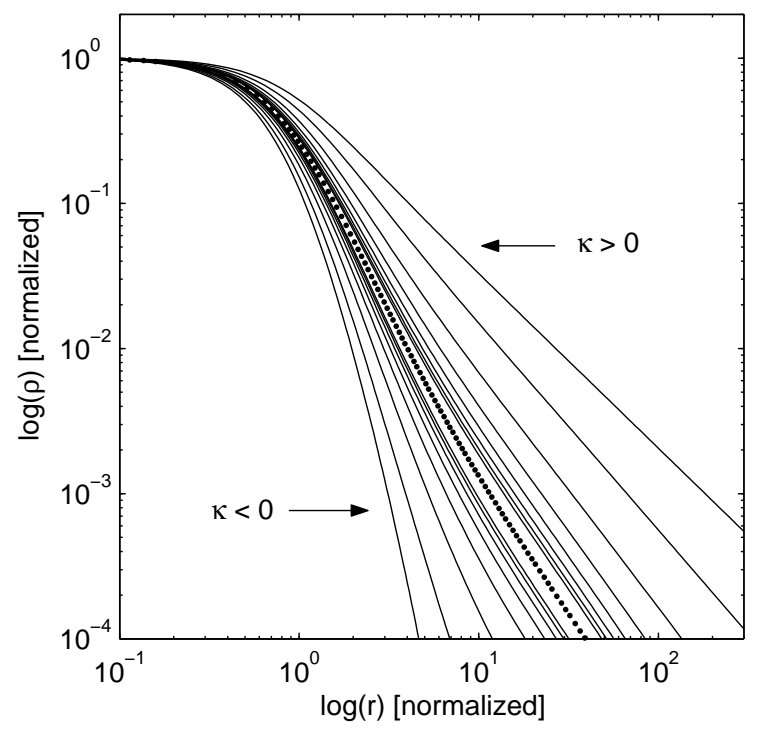

Fig. 1. - Nonextensive density profiles: for $\kappa=3$ the innermost and outermost curves correspond to the $\rho^{-}$and $\rho^{+}$solutions, respectively. For increasing $\kappa=4 \ldots 10$ both sets of curves converge to the central isothermal sphere solution $(\kappa=\infty)$ indicated by dots, $\rho^{-}$from inside and $\rho^{+}$from outside.

$\Delta \Phi=4 \pi G \rho$ reads

$$
\Delta \Phi=4 \pi G \int f\left(\frac{1}{2} v^{2}+\Phi\right) d^{3} v,
$$

and represents the fundamental equation governing the equilibrium of the system, where $f\left(\frac{1}{2} v^{2}+\Phi\right)=f(E)$ depends on the energy only. Commonly, the relative particles energy $E_{r}=-1 / 2 v^{2}+\Psi$ is introduced where $\Psi$ is the relative potential $\Psi=-\Phi+\Phi_{0}$, chosen to vanish at the systems boundary and satisfying Poisson's equation as $\Delta \Psi=-4 \pi G \rho$.

If $f\left(E_{r}\right)$ resembles the exponential mass distribution function defining the structure of an isothermal self-gravitating sphere of gas, in this case identical to the phase-space density distribution of a collisionless system of particles,

$$
f\left(E_{r}\right)=\frac{\rho_{0}}{\left(2 \pi \sigma^{2}\right)^{3 / 2}} \exp \left(-\frac{v^{2} / 2-\Psi}{\sigma^{2}}\right)
$$

then the corresponding density distribution $\rho=$ $\rho_{0} \exp \left(\Psi / \sigma^{2}\right)$ is found after integrating over all ve- locities. Combining with Poisson's equation (2) the solution governs the structure of the isothermal self-gravitating sphere (Binney \& Tremaine 1994). The equilibrium distribution Eq. (3) can be obtained by extremizing the standard BGS entropy with regard to conservation of mass and energy.

Since Eq. (3) applies exclusively to a system of independent particles we introduce now long-range interactions by the generalized entropy functional (1). Extremizing Eq. (1) after replacing the entropy function flnf of an uncorrelated ensemble by the generalized functional $-\kappa f\left(1-f^{1-1 / \kappa}\right.$ and applying Lagrange multipliers (Plastino \& Plastino 1993) the resulting distribution function reads

$$
f^{ \pm}\left(E_{r}\right)=B^{ \pm}\left[1+\frac{1}{\kappa} \frac{v^{2} / 2-\Psi}{\sigma^{2}}\right]^{-\kappa}
$$

As previously, the superscripts refer to the positive or negative intervals of the entropic in$\operatorname{dex} \kappa$, accounting for less $(+)$ and higher (-) organized states and thus reflecting the accompanying entropy increase or decrease, respectively. If we identify $f^{ \pm}\left(E_{r}\right)$ again as mass distribution, the $\kappa$-dependent generalized constants $B^{ \pm}$ assure proper normalization and dimension and differ for positive and negative definite $\kappa$-values as $B^{+}=C \Gamma(\kappa) /\left(\kappa^{3 / 2} \Gamma(\kappa-3 / 2)\right)$ and $B^{-}=C \Gamma(\kappa+$ $5 / 2) /\left(\kappa^{3 / 2} \Gamma(\kappa+1)\right)$, where $C=\rho_{0} /\left(2 \pi \sigma^{2}\right)^{3 / 2}$ and $\Gamma$ denotes the standard gamma function (Leubner 2004). The different normalization is caused by the interval corresponding to negative $\kappa$-values, which generates an energy cutoff in Eq. (4) leading to the constraint $v^{2} / 2-\Psi \leq \kappa \sigma^{2}$ and restricting also the integration limits in velocity space. For $\kappa \rightarrow \infty$ Eq. (4) approaches the exponential distribution function (3) defining the density profile of the isothermal sphere.

After incorporating the sign of $\kappa$ into Eq. (4), we perform separately for positive and negative definite $\kappa$ the integration of (4) over all velocities where $B^{ \pm}$must be used consistently. The resulting solution provides a modification of the velocity space context introduced by Leubner (2004) for the density evolution of a system in gravitational potential as

$$
\rho^{ \pm}=\rho_{0}\left[1-\frac{1}{\kappa} \frac{\Psi}{\sigma^{2}}\right]^{3 / 2-\kappa}
$$




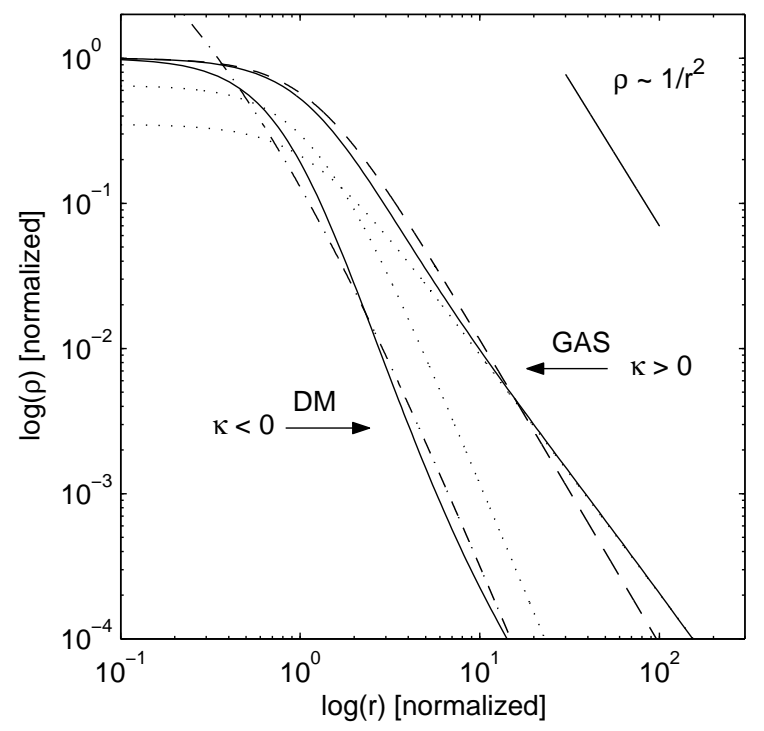

Fig. 2.- Comparison of the DM nonextensive density profile $(\kappa=-7, \sigma=1$, solid $)$ with the NFW-profile $\left(r_{s}=0.65, \rho_{0}=1.2\right.$, dasheddotted). The radial nonextensive gas distribution $(\kappa=7)$ is compared with a single $\beta$-model ( $\beta=0.7$, dashed $)$ and the decomposition of a double $\beta$-model $\left(\beta_{\text {core }}=0.98, \beta_{\text {halo }}=0.55\right.$, dotted $)$. As illustration a dependence $\rho \simeq 1 / r^{2}$ is added.

In analogy to the corresponding tandem character in velocity space Eq. (5) generates in a gravitational potential for finite positive values of $\kappa$ pronounced density tails, whereas for negative $\kappa$ values the solutions are restricted within the cutoff at $\kappa=\Psi / \sigma^{2}$ and $\kappa=-\infty$.

The duality of equilibria in nonextensive statistics is manifest in two families, the nonextensive thermodynamic equilibria and the equilibria of kinetic equations, where both are related by $q^{\prime}=2-q($ Karlin, Grmela \& Gorban 2002). Since $q=1-1 / \kappa$ (Leubner 2002) we find for the entropic index $\kappa^{\prime}=-\kappa$ relating the two families of equilibria, where with regard to Eq. (5) $\kappa>0$ corresponds to the stationary states of thermodynamics and $\kappa<0$ to kinetic stationary states. The limiting BGS state for $\kappa=\infty$ is therefore characterized by self-duality. The nonextensive parameter $\kappa$ finds also a physical interpretation in terms of the heat capacity of a medium (Almeida 2001). A system with $\kappa>0$ represents an environment with finite positive heat capacity and vice versa, for $\kappa<0$ the heat capacity is negative. Negative heat capacity is a typical property of self-gravitating systems, see e.g. Firmani et al. (2000). Moreover, contrary to thermodynamic systems where the tendency to dis-organization is accompanied by increasing entropy, self-gravitation tends to result in higher organized structures of decreased entropy. Consequently, the nonextensive bifurcation of the singular isothermal sphere solution into two distributions $f^{ \pm}\left(E_{r}\right)$ or $\rho^{ \pm}$, respectively, requires to identify the density profile (5) for positive definite $\kappa$ as the proper distribution of the thermodynamic state of the gas, whereas the negative definite counterpart is associated to the selfgravitating DM distribution. For $\kappa \rightarrow \infty$ both solutions merge at the isothermal sphere distribution defined by Eq. (3).

After elucidating the tandem character of the nonextensive context we combine Poisson's equation $\Delta \Psi=-4 \pi G \rho$ with Eq. (5) to find a second order nonlinear differential equation for for the radial density dependence of a spherically symmetric gas and DM distribution as

$$
\begin{array}{r}
\frac{d^{2} \rho}{d r^{2}}+\frac{2}{r} \frac{d \rho}{d r}-\left(1-\frac{1}{n}\right) \frac{1}{\rho}\left(\frac{d \rho}{d r}\right)^{2}- \\
\frac{4 \pi G n}{(3 / 2-n) \sigma^{2}} \rho^{2}\left(\frac{\rho}{\rho_{0}}\right)^{-1 / n}=0
\end{array}
$$

where $n=3 / 2-\kappa$ is introduced. n corresponds to the polytropic index $n=1 /(\gamma-1)$ of stellar dynamical systems where $\gamma$ is the adiabatic index. Hence, the limit $\kappa=\infty$ is governed consistently with $\gamma=1$ by the equation of state of the isothermal sphere (Binney \& Tremaine 1994) and Eq. (4) denotes the nonextensive generalization. Multiplying Eq. (7) in the limit $\kappa \rightarrow \infty$ by $r^{2} / \rho$ and combining the first three terms on the left hand side as $d / d r\left(r^{2} d l n \rho / d r\right)$ recovers the equation defining the conventional isothermal sphere. Eq. (7) is solved numerically by simulation of the corresponding two first order differential equations.

\section{Results and discussion}

As natural consequence of nonextensive entropy generalization the isothermal sphere profile bifurcates into two distribution families controlled by the sign of $\kappa$ and governed by Eq. (7). The self- 


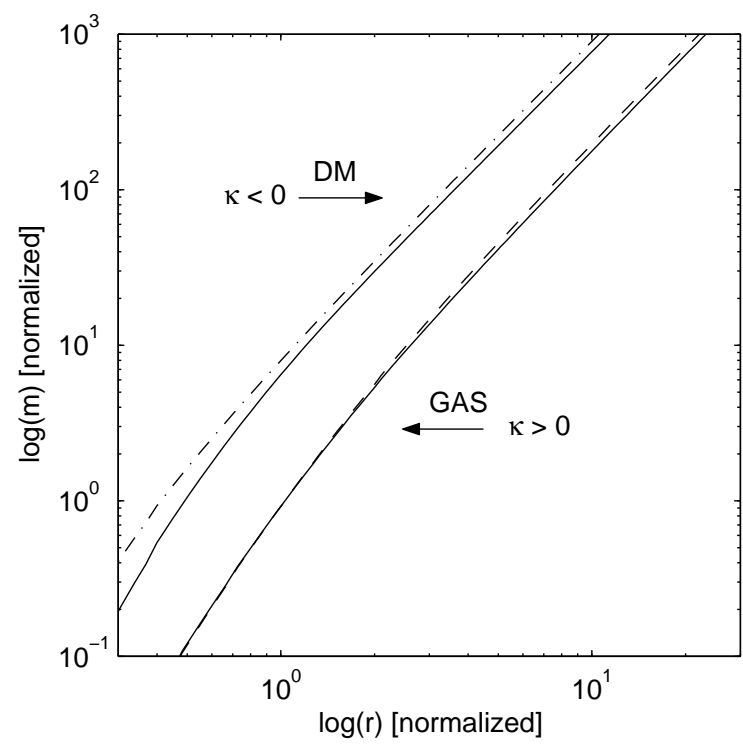

Fig. 3.- Nonextensive integrated mass of DM and gas components (solid) as compared with the NFW-profile (dashed-dotted) and the single $\beta$-model (dashed) for the gas component. Parameters are the same as for Fig. 2.

gravitating DM component, a lower entropy state due to gravitational interaction, resides besides a thermodynamic gas component of increased entropy. Fig. 1 provides the radial density profile characteristics and $\kappa$ dependences as computed from Eq. (7) for both families, DM distributions located below and the plasma distributions above the limiting exponential solution. With increasing entropic index $\kappa$ both braches merge simultaneously in the isothermal sphere density profile, since the power-laws (4) converge for $\kappa \rightarrow \infty$ to the exponential energy distribution (3), corresponding to $n=\infty$ in Eq. (7). Physically finit values of $\kappa$ represent long-range interactions and correlations within the system, whereas the transition to $\kappa=\infty$ in the central curve defines the extensive limit of statistical independence. Since we focus here on the shapes of radial profiles and their physical foundations, normalizations are conveniently applied. As $r \rightarrow 0, \rho=\rho_{0}=1$ and the solution meets the physically required condition $d \rho / d r=0$ in the origin.

In Fig. 2 we compare one negative (DM) solution to Eq. (7) with the NFW model as well as one symmetrically, positive (gas) solution with a single $\beta$-model. On small scales the theoretical DM density distribution is characterized consistently with e.g. Firmani et al. (2000) by a shallow core of finite density as $r \rightarrow 0$. Despite a gradually tail-like structure of the theoretical profile the halo characteristics are similar to the NFW profile. Changes in the mean energy or variance $\sigma$ generates a radial shift of the entire profile, which practically corresponds to variations of the scale radius $r_{s}$ of the NFW model. As measure of the long-range interactions the dimensionless second parameter $\kappa$ of the theory controls the shape and characteristic mean slope of the profile on intermediate and large scales, see also Fig. 1. Thus both, shallower or steeper slopes (Moore et al. $1998,1999)$, interpreted in the nonextensive context as consequence of different coupling strengths, are accessible. Recently an improved fitting formula, converging to a finite density in the center, was introduced and compared with the standard NFW-profile as well (Navarro 2004). The accompanying discussion illustrates critically also the potential difficulties arising in simulations of the innermost structure of CDM haloes (see also Trott \& Melatos (2005)). On the other hand, highresolution rotation curve analyses of galaxies are consistent only with cored haloes (Blok et al. 2003; Gentile et al. 2004) supporting the physical consequences of the nonextensive statistics formulation from observations. The nonextensive gas density distribution follows a single $\beta$-model in the core but deviates by a halo tail formation. This deviation can be accurately fitted by a double $\beta$-model (a decomposition as in Xue \&Wu (2000) is shown by the dotted lines), indicating that the nonextensive theory provides naturally a theoretical context able to solve the $\beta$-discrepancy.

Fig. 3 presents the radial dependence of the integrated mass of DM and gas components for symmetric values of $\kappa= \pm 7$ as compared to the NFW and $\beta$-model, where on observational grounds a $20 \%$ central gas density fraction $\rho_{0}$ is introduced for proper visualization. Consistent with Fig. 2 the NFW integrated mass exceeds the nonextensive solution slightly, whereas the integrated $\beta$-model is practically identical to the generalized entropy approach.

The dual nature of the nonextensive theory provides a solution to the problem of DM and gas density distributions of clustered matter from fun- 
damental physics, where both parameters $(\kappa, \sigma)$ admit physical interpretation. The bifurcation of the density distribution into a kinetic DM and thermodynamic gas branch turns out as natural consequence of the theory and is controlled by the entropic index $\kappa$, accounting physically for nonlocality and long-range interactions in nonextensive systems. Due to different correlation properties of clustered structures, particular DM and gas density profiles might be subject to different values of $\kappa$ and $\sigma$, regulating details of possibly non-universal, mass dependent profiles. The theory was found to reproduce accurately also density profiles generated by N-body and hydrodynamic simulations, a subject to be addressed elsewhere. In conclusion, it is proposed to favor the family of nonextensive distributions, derived from the fundamental physical context of entropy generalization, over empirical models when fitting observed density profiles of astrophysical structures.

\section{REFERENCES}

Almeida, M. P. 2001, Physica A 300, 424

Andrade, J. S., Almeida, M. P., Moreira, A. A., \& Farias, G. A. 2002, Phys. Rev. E 65, 036121

Bahcall N. A., \& Lubin, L. M. 1994, ApJ, 426, 513

Binney, J. \& Tremaine, S. 1994, Galactic Dynamics, Princeton Univ. Press.

Blok, W. J. G., Bosma, A., \& McGaugh, S. 2003, MNRAS, 340, 657

Borriello, A. \& Salucci, P. 2001, MNRAS, 323, 285

Burkert, A. 1995, ApJ, 447, L25

Burkert, A. 2000, ApJ, 534, L143

Cavaliere, A., \& Fusco-Femiano, R. 1976, A\&A 49,137

Firmani, C., Onghia, E. D., Avila-Reese, V., Chincarini, G., \& Hernandez, X. 2000, MNRAS 315, L29

Fukushige, T., \& Makino, J. 1997, ApJ 477, L9

Gentile, G., Salucci, P., Klein, U., Vergani, D., \& Kalberla, P. 2004, MNRAS 351, 903

Jing, Y. P. \& Suto, Y. 2000, ApJ,529, L69
Kaniadakis, G., Lavagno A., \& Quarati, P. 1996, Phys. Lett. B 369, 308

Kaniadakis G. 2002, Phys. Rev.E 66, 056125

Karlin, I. V., Grmela, M., \& Gorban, A. N. 2002, Phys. Rev. E 65, 036128

King, I. R. 1967, Astron. J., 67, 471.

Leubner, M. P., \& Schupfer, N. 2001, J. Geophys. Res. 106, 12993

Leubner, M. P. 2002, Ap\&SS 282, 573

Leubner, M. P. 2004, ApJ 404,469

Leubner, M. P., \& Voeroes, Z. 2005, ApJ 618,547

Milovanov, A. V., \& Zelenyi, L. M. 2000, Nonlin. Proc. Geophys. 7, 211

Merritt, D., Navarro, J. F., Aaron, L., \& Jenkins, A. 2005, ApJ, 624, L85

Moore, B., Governato, F., Quinn, T., Stadel, J., \& Lake, G. 1998, ApJ, 499, L5

Moore, B., Quinn, T., Governato, F.,Stadel, J., \& Lake, G. 1999, MNRAS 310, 1147

Nakamichi, A., Joichi, I., Iguchi, O., \& Morikawa, M. 2002, Chaos, Solitons \& Fractals 13, 595.

Navarro, J. F., Frenk, C. S., \& White, S. D. M. 1996, ApJ 462, 563

Navarro, J. F., Hayashi, E., Power, C., Jenkins, A. R., Frenk, C. S., White, S. D. M., Springel, V., Stadel, J., \& Quinn, T. R. 2004, MNRAS 349, 1039

Navarro, J. F., Frenk, C. S., \& White, S. D. M. 1997, ApJ 490, 493

Ota, N. \& Mitsuda, K. 2004, A \& A, 428, 757

Plastino, A. R., \& Plastino, A. 1993, Phys. Lett. A 174,384

Plastino, A. R., Plastino, A., \& Tsallis, C. 1994, J. Phys. A: Math. Gen. 27, 5707

Renyi, A. 1955, Acta Math. Hungaria, 6, 285

Ricotti, M. 2003, MNRAS, 344, 1237

Salucci, P., \& Burkert, A. 2000, ApJ 537, L9 
Silva, R., Plastino, A. R., \& Lima, J. A. S. 1998, Phys. Lett. A 249, 401

Spergel, D. N. \& Steinhard, P. J. 2000, PRL 84, 3760

Trott, C. M., \& Melatos, A. 2005, ApJ 618, 38

Treumann, R. A. 1999, Physica Scripta 59, 19

Tsallis, C. 1988, J. Stat. Phys. 52, 479

Tsallis, C. 1995, Physica A 221, 277

Xue, Y-J., \& Wu, X-P. 2000, MNRAS, 318, 715

Zhao, H. 1996, MNRAS, 278, 488

This 2-column preprint was prepared with the AAS LATEX

macros v5.2. 